\title{
Analysis of An End-to-End Proportional Bandwidth Allocation Algorithm
}

\author{
L. Huynh (*), A. Nilsson (**) \\ (*) IBM Networking Systems \\ P.O. Box 12195, Research Triangle Park, NC, 27709, U.S.A. \\ laph@vnet.ibm.com \\ phone: (919) 254-4386, fax: (919) 254-4975 \\ (**) Electrical and Computer Engineering Department \\ North Carolina State University \\ P.O. Box 7911, Raleigh, NC. 27695, U.S.A.
}

\begin{abstract}
In this paper, we present a new proportional bandwidth allocation scheme along with its analysis. The novel approach of this scheme is that it is purely end-to-end, it does not require any processing by the network, it is adaptive to changing network conditions, it has minimum oscillations, and it works even when sources have different end-to-end propagation delays. This scheme is a function of an Adaptive RateBased (ARB) congestion control algorithm that is designed to support best-effort traffic for which the proportional bandwidth allocation function is very desirable. We will briefly describe the ARB algorithm. We then analyze the behavior of the bottleneck queue, the settings of the ARB parameters and their effects on the behavior. This analysis will lead us to two conditions that, when they are satisfied, will provide proportional bandwidth allocation among the sources that are controlled by the ARB algorithm. We will show what parameters and how they can be controlled in order to achieve the proportional bandwidth allocation. Simulation will be used to validate our analysis.
\end{abstract}

\section{Keywords}

Adaptive rate-based congestion control, proportional bandwidth allocation, performance analysis 


\subsection{INTRODUCTION}

Supporting best-effort traffic has become increasingly important in highspeed network. Recently, the ATM Forum has adopted an end-to-end rate-based feedback congestion control mechanism for this traffic class, also referred to as the Available Bit Rate (ABR). The reason for its importance is that this class of traffic can maximize the overall network efficiency by fully utilizing the available bandwidth in the network. One characteristic that makes this class of traffic desirable is that it can tolerate variations in the delay and bandwidth that the network can provide. But this tolerance also comes with a requirement in that the network must assure fairness in the amount of bandwidth allocated to all the connections of this traffic class, that share a network path or a bottleneck link in the network. With respect to this fairness, rather than allocating equal bandwidth to all connections, it is more desirable to assign the bandwidth based on the demand of each connection. If a connection that has twice the amount of traffic load compared with others, it should be assigned twice the amount of bandwidth when the network/path is fully utilized. We refer to such a scheme as a proportional bandwidth allocation scheme. Clearly, since best-effort traffic has to adapt to the availability of bandwidth in the network, some feedback signal that reflects the network conditions is necessary for the sources of this traffic class to control their respected bandwidth. This naturally leads to the need to have an efficient congestion control mechanism that can also provide proportional bandwidth allocation function. Recent proposals only include schemes that require extensive processing by the network (see Huynh, 1996 for a latest list of references) to provide this function. The algorithm we propose here will achieve this without any requirement on the network.

On the issue of congestion control, it has always been a focus of research and development since the initial days of computer networking. The objectives are to make more efficient use of network resources (e.g., buffer, bandwidth etc.), to enforce fairness among users, and to provide some guarantee in terms of service qualities (e.g., response time, throughput). A better congestion control mechanism would yield better throughput-cost ratio without sacrificing other objectives.

Congestion control mechanisms can basically be classified into openloop and closed-loop schemes. Open-loop schemes require resources (i.e., bandwidth, buffers etc.) to be reserved ahead of time, whereas closed-loop implies a feedback mechanism. That is information about the status of the network is fed back to the sources of traffic, which will take actions according to these information. Clearly, the objective of these feedback mechanisms is to maximize the network efficiency by allowing sources under their control to utilize the unused bandwidth while, at the same time, minimizing loss of data. Extensive research has been done in the area of feedback congestion control mechanisms, especially recently for high-speed networks (ATM, 1996; Haas, 1991; Jacobson, 1988; Jain, 1989; Keshav, 1991; Kim, 1995; Kolarov, 1995; Mitra, 1991; Rose, 1992; to name but a few). In this paper, we describe 
a new end-to-end Adaptive Rate-Based Congestion Control (ARB) mechanism that is designed based on the principles of rate control and preventive mechanism, to meet the following objectives: the algorithm is simple to implement; it is adaptive to network conditions and responsive to the user offered load; it maximizes throughput and minimizes congestion; and finally, it provides proportional bandwidth allocation function. This algorithm has been implemented in the new IBM's high performance packet routing network.

The rest of this paper is structured as follows. In section 2, we give a brief description of the algorithm. The unique feature that allows it to control the proportional bandwidth of each connection is emphasized. We then discuss the stability of the algorithm. In section 3 , we present the time dependent queue analysis that takes into account the statistical variations of the arrival process, to study the behavior of the bottleneck queue and the effect of the ARB parameters on it. Based on this analysis, we propose two conditions that, when they are satisfied, will provide proportional bandwidth allocation among the connections that are controlled by the ARB algorithm. In section 4, we show the worst case throughput allocation when one or both of the conditions are not satisfied. In section 5, we present the results of our numerical and simulation study. In section 6 , we discuss some future research topics.

\subsection{THE ARB ALGORITHM}

The ARB algorithm employs a closed-loop distributed control mechanism based on periodic exchange of information between two endpoints of a full-duplex connection (with respect to ATM, two virtual channels make up a full duplex connection between two end-points). Each connection end-point, therefore, consists of two components, a sender and a receiver that cooperate to manage the connection. Periodically, the sender sends a sampling cell/packet that contains the elapsed time since its previous sampling cell (note that the sampling cell can be piggy-backed with data and not a stand-alone cell). The receiver based on its sampling cell derives the current delay along the path in the network and together with the delay information it maintains for the path, it derives an action (NORMAL, RESTRAINT, SLOWDOWN1, or SLOWDOWN2) to be taken by the sender. The actions are derived based on three thresholds $T_{1}, T_{2}$, and $T_{3}$ that are assigned to a connection based on the bottleneck link along the path of that connection. The normal operation of an ARB connection involves mainly three actions, NORMAL, RESTRAINT, and SLOWDOWN1 respectively (e.g., excessive delay, out of normal operating range) condition and to force ARB connections to react quickly. This overload condition can be caused when new connections are started in an already saturated network (or on a path), or sudden bursts of higher priority traffic (e.g., delay sensitive traffic) etc.. The sender, based on the information returned by the receiver, regulates the input traffic load accordingly. We use the phase, 'sampling cell/packet', to show that the ARB algorithm can be employed either at a lower layer (e.g., ATM adaptation layer), or 
at the transport layer (e.g., TCP). In fact, a version of $A R B$ has been implemented in a new transport protocol at IBM.

We define the following parameters in order to describe the sampling algorithm. Let $M s_{n}$ be the length of the $n$th sampling interval at the sender, let $M r_{n}$ be the length of the corresponding interval observed at the receiver, and let $d_{n}$ be the change in network/path delay observed at the $n$th sampling epoch. Let $D_{n}$ be the accumulated delay observed at the receiver up to the $n$th sampling epoch since the last rate reduction, and $D_{\text {accum }}$ is the total accumulated delay maintained at the receiver. Figure 1(a) shows the sampling mechanism of ARB. The path delay at the $n$th sampling epoch is computed as follows (note that $M s_{n}$ is provided by the sender along with the sampling request): $d_{n}=$ $M r_{n}-M s_{n}$. When $d_{n}>0$, it is an indication that there is a queue build-up that is currently taking place in the network. When $d_{n}=0$, it is an indication that there is no additional queue build-up since the last sampling request. And when $d_{n}<0$, it is an indication that there is less queue build-up since the last sampling request. $D_{n}$ is computed as: $D_{n}=\sum_{i}^{n} d_{i}$, where $\mathrm{k}$ represents the last epoch when $D_{k}$ was reset to zero cbiresponding to a rate reduction at the sender (SLOWDOWN1). Please refer to (Huynh, 1996) for a detailed description of the algorithm, including the use of $D_{\text {accum }}$.

One important feature of $A R B$ is that it minimizes the oscillation in the send rate (i.e., throughput) of a connection. This is accomplished by distributing the rate reduction to all connections once the queue exceeds the $T_{2}$ threshold (i.e., a source cuts its send rate by a SLOWDOWN1 factor when its sampling cell/packet detects that the queue has exceeded this threshold). Without this feature, a source may be forced to cut more times than others due to statistical behavior of the bottleneck queue with respect to the arrival of the source's sampling cells. Consequently, that source's send rate will be reduced significantly relatives to others and it takes a long period for it to reclaim its share of the bandwidth. As a result, proportional bandwidth allocation can not be enforced over any reasonable amount of time.

Analysis in proving the stability of a general feedback congestion mechanism with linear increase and multiplicative decrease has recently been studied in (Altman, 1994), (Keshav, 1991), (Mukherjee, 1994). ARB belongs to this class of algorithm. Also in (Artiges, 1994), the author analytically proves the stability of the ARB algorithm based on a single source model.

The average send rate of connection $i, A_{i}(i=1, \ldots, N ; N$ is the total number of connections), at steady state is given in the following equation (derived by noting that at steady state, the send rate of connection i fluctuates between the upper value and the lower with respect to the cycle of NORMAL, RESTRAINT, and SLOWDOWN1 actions respectively; see Huynh, 1996):

$$
A_{i}=\Delta I_{i} \frac{P_{i}(I)}{P_{i}(D)} \frac{1}{\theta_{1}}\left[\frac{2-\theta_{1}}{2}\right] .
$$


Where $\Delta /$ is the rate increment amount assigned to connection i. $\theta_{1}$ is the rate reduction factor corresponding to SLOWDOWN1. $\frac{P_{i}(l)}{P_{i}(D)}$ is the ratio of the probability of a sampling cell that results in an incierse over the probability of a sampling cell that results in a decrease, both of connection i. By keeping $\theta_{1}$ and $\frac{P_{i}(l)}{P(D)}$ the same for all connections, $\Delta l_{i}$ becomes a simple parameter $P$ hif $(9)$ can be manipulated to assign different proportional throughputs for different connections. The same $\theta_{1}$ can easily be assigned to all the connections, whereas the $\frac{P_{i}(l)}{\left.P_{(}\right)}$ratio depends on other parameters of the algorithm. That is the toff(R) of our analysis to follow.

\subsection{ANALYSIS}

One important objective of the analysis is to study the behaviors at the bottleneck queue, that enable the ARB algorithm to provide the function of proportional bandwidth allocation. We only provide a summarized version here, please refer to (Huynh, 1996) for details.

First, we define the following assumptions for the model: 1) cells are served at the bottleneck queue under FCFS service discipline; 2 ) sampling cells from all sources arrive uniformly at the bottleneck queue; 3 ) all sources behave according to the same ARB algorithm but can have different parameters; 4 ) all sources always have traffic to send. The last assumption is the situation when proportional bandwidth allocation is desirable (sources are allocated different throughput and use all of what they're allowed). It is also the traffic pattern that is commonly agreed to be a real test for a congestion control algorithm.

We will begin our analysis of the bottleneck queue with the diffusion approximation model (Newell, 1982). Figure 1(b) shows the simplified queueing model for our analysis.

Let $Q(t)$ be the bottleneck queue length at time $t$, and $F(q, t)=P\{Q(t) \leq q\}$ be the distribution of the queue at time t. Based on the diffusion approximation, $F(q, t)$ satisfies the following equation:

$\frac{\partial F(q, t)}{\partial t}=-a(t) \frac{\partial F(q, t)}{\partial q}+\frac{b(t)}{2} \frac{\partial^{2} F(q, t)}{\partial q^{2}}$.

Where $a(t)$ is the instantaneous rate of change in the expected queue length and $b(t)$ is the instantaneous rate of change in the variance of the queue length at the bottleneck respectively. Let $\lambda(t)$ be the total arrival rate at time $t$ of all the connections that send data through this bottleneck queue and are sensitive to its behavior, and $\mu(t)=\mu$ be the service rate at time $t$ that serves traffic from these connections. Then from the above, we have $a(t)=\lambda(t)-\mu$. Our emphasis here is to study the behavior of the queue and so the time is chosen relative to the 


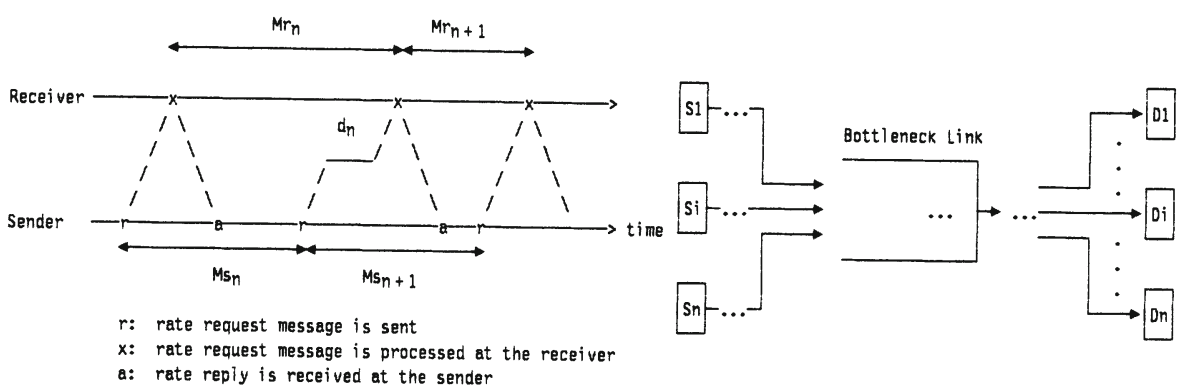

(a)

(b)

Figure 1. Rate Measurement and analysis model.

queue only. We will include propagation delay later when necessary to take into account the delayed reaction of sources.

With respect to $b(t)$, since the departure process is deterministic, we need to study the variance of the number of arrivals as a function of time (the variance of the queue length is the sum of the variance of the arrival process and the departure process). To approximate this term, we now use discrete counting process to study the variance of the arrival process. From earlier assumption, our model is such that sources always have traffic to send. Let $C(t)$ be the number of arrivals up to time $t$. Let $s_{i}$ be the average period of source ith in this interval $(0, t]$. As a result of the arrival process, $C(t)$ will consist of a deterministic part and a random part, let's refer to the random part as $R(t)$.

Let's now consider the number of arrivals generated by a source. At a given period $s_{i}$, source $i$ will generate $\mathrm{I}\left(\frac{t}{s_{j}}\right)$ cells where $\mathrm{I}(x)$ is the integer operation of $x$ (i.e., the floor operation). In addition, source i can generate one more cell in this time interval with the following probability:

$$
z_{i}=\frac{t}{s_{i}}-\mathrm{I}\left[\frac{t}{s_{i}}\right]
$$

Assuming that $t$ is uniformly distributed over source i's period $s_{i}$. The contribution to the random number of arrivals from each source is a binomial distribution with probability $z_{i}$. The variance of this random arrival from each source is clearly $z_{i}\left(1-z_{i}\right)$. Assuming independence between sources, the total variance due to the random arrival is:

$\operatorname{Var}(R(t))=\sum_{i=1}^{N} z_{i}\left(1-z_{i}\right)$.

Where $\mathrm{N}$ is the number of connections that directly compete for bandwidth at a bottleneck link. Clearly, this variance is bounded at $\operatorname{Var}(R(t)) \leq B^{2}=\frac{1}{4} N$ that is independent of $t$. As a result, we can bound the variance of the continuous time arrival process and simplify eq. (2) with $b(t)=0$ (note that the departure process is deterministic and since 
the server is always busy, its variance is zero). Therefore, the solution of eq. (2) for the queue length at the bottleneck as a function of time is thus:

$$
\frac{d F\left(q, t \mid q_{0}, t_{0}\right)}{d q}=\frac{1}{\sqrt{2 \pi} B} e\left[-\frac{1}{2}\left[\frac{q-q_{0}-m\left(t, t_{0}\right)}{B}\right]^{2}\right] .
$$

Where $m\left(t, t_{0}\right)=\int_{t_{0}}^{t} a(u) d u$. The bottleneck queue behaves in a cyclic manner based on the behavior of the ARB sources. Each cycle contains three separate stages: the queue behavior during the rate increasing stage, the queue behavior during the rate restraining stage, and finally, the queue behavior during the rate decreasing stage. Eq. (3) allows us to study the upperbound on the stages of the queue, that are summarized in the following.

\subsection{The Queue Behavior During The Increasing Stage}

When the total arrival rate $\lambda(t)$ is below the bottleneck rate $\mu$, ARB sources will increase their send rates. As $\lambda(t)$ approaches $\mu$, the queue starts to grow. Let's anchor the initial time (i.e., $t_{0}=0$ ) at the point where the queue starts to grow. Let $\lambda_{0}$ be the total rate at $t_{0}$ and let $q_{0}=0$. The mean queue length will grow as follows:

$m(t)=\frac{1}{2} \alpha t^{2}+\left(\lambda_{0}-\mu\right) t$.

$\alpha$ is the rate increasing factor as described in the appendix. The queue will continue to grow until it exceeds $T_{1}$ threshold where $\lambda(t)>\mu$ and the Restraining Stage is entered. Note that when the mean queue length exceeds $T_{1}+B$, it is unlikely for any source to increase since the mean queue length begins to dominate the threshold level $T_{1}$, i.e., $F\left(T_{1}, t \mid q_{0}=0, t_{0}=0\right) \rightarrow 0$. Let $P_{1}$ be the length of the increasing stage, and $Q$, be the queue length at the end of this stage, then they can be approximated as follows, assuming that $\lambda_{0} \simeq \mu$ :

$$
\begin{aligned}
& P_{1} \simeq \sqrt{\frac{2\left(T_{1}+B\right)}{\alpha}}+r t t . \\
& Q_{1} \simeq \frac{1}{2} \alpha\left[\sqrt{\frac{2\left(T_{1}+B\right)}{\alpha}}+r t t\right]^{2} .
\end{aligned}
$$

Where $r t$ is the weighted average of the roundtrip times from all the sources sharing the bottleneck link. Clearly, the send rate right before the restraining stage is entered is larger than the bottleneck service rate as the queue continues to grow. This is the maximum total arrival 
rate to the bottleneck queue, $\lambda_{\max }$ it can be computed to be: $\lambda_{\max }=\lambda_{0}+\alpha P_{1}$.

\subsection{The Queue Behavior During The Restraining Stage}

During this stage, we have $\lambda_{\max }>\mu$ and sources are restrained from increasing their rate, the corresponding bottleneck queue continues to grow until it exceeds the $T_{2}$ threshold. Again, let's anchor the relative time $t_{0}=0$ at the beginning of the restraining stage. Note that the initial queue length for this period is: $q_{0}=Q_{1}$ from the analysis of the increasing stage. The growth in the mean queue length during this period where $t>t_{0}$ is simply:

$m(t)=\left(\lambda_{\max }-\mu\right) t \quad$ for $t>t_{0}$.

When the mean queue length exceeds $T_{2}+B$, it is unlikely for any source to remain restraint since the mean queue length begins to dominate the threshold level $T_{2}$, i.e., $F\left(T_{2}, t \mid q_{0}=Q_{1}, t_{0}=0\right) \rightarrow 0$. Let $P_{R}$ be the length of the period of the restraining stage, and $Q_{R}$ be the queue length at the end of this stage, they can be derived as follows:

$P_{R}=\frac{\left(T_{2}+B-Q_{1}, 0\right)^{+}}{\lambda_{\max }-\mu}+r t+t_{s}$

$Q_{R}=\left(\lambda_{\max }-\mu\right) P_{R}+Q_{1}$

Where $(x, y)^{+}=\max (x, y)$, and $t_{s}$ is the length of time until the arrival of the next sampling cell. The reason for this is that sources will only detect the condition of the queue exceeding $T_{2}+B$ when their sampling packets arrive at the queue after the occurrence of this condition ( $\left.F\left(T_{2}, t\right) \rightarrow 0\right)$. The roundtrip time is the delay until the effect of rate cutting is observed at the queue.

\subsection{The Queue Behavior During The Decreasing Stage}

During this stage, a source that detects the queue exceeding the $T_{2}$ threshold will cut its send rate by a factor of $\theta_{1}$. This process continues until the bottleneck queue goes below the $T_{2}$ threshold that is when sources will restraint from further cutting. The queue continues to decrease due to the fact that the send rate is less than the service rate (i.e., $\lambda(t)<\mu$ ). The cycle resumes when the queue goes below the $T_{1}$ threshold and sources start to increase again. The period during which $t$ he queue begins to decrease until it reaches zero can be split into two sub-periods: one is the period where the queue decreases as the sources decrease their send rates; the other is the period where the queue continues to decrease (as a result of the total send rate being less than the bottleneck service rate) toward zero but the sources have stopped decreasing. Let $P_{D}$ be the total decreasing period, and $P_{D d}$ and 
$P_{D,}$ be the corresponding sub-periods respectively. We will now derive the length of each sub-period.

Let's again anchor the initial time when the decreasing stage starts to be 0 , the reduction in the mean queue length at time $t>t_{0}$ during this period is:

$m(t)=\lambda_{\max }\left[\frac{\beta^{t}-1}{\ln \beta}\right]-\mu t$.

The initial queue for this period is $q_{0}=Q_{R}$. The queue continues to grow beyond $Q_{R}$ for as long as the total arrival rate that is on the way down is larger than the server's rate. We can compute the subperiod $P_{D d}$ to be:

$$
\begin{array}{ll}
P_{D d}=\left(t_{D d}+t^{\prime}, M s_{\max }\right)^{-} & \text {such that } Q_{R}+m\left(P_{D d}\right) \geq 0 \\
& \text { and } 0 \leq t^{\prime} \leq r t t .
\end{array}
$$

Where $t_{D d}$ can be computed from: $m\left(t_{D d}\right)=\left(T_{2}-B, 0\right)^{+}-Q_{R} . M s_{\max }$ is the maximum sampling interval of all the sources. The queue length, $Q_{D d}$, at the end of this $P_{D d}$ subperiod is: $Q_{D d}=Q_{R}+m\left(P_{D d}\right)$.

Once sources restraint from further cutting, the queue continues to decrease linearly since the total arrival rate is below the server's rate. The time until the queue becomes zero is (following the restraining stage analysis):

$$
P_{D r}=\frac{-Q_{D d}}{\left(\lambda_{\min }-\mu\right)} .
$$

The total time during the decreasing stage is therefore: $P_{D}=P_{D d}+P_{D r}$.

From the above analysis, the following values are derived: $R I$, the rate increasing period; $C T$, the time for the queue to go through a complete cycle from the beginning of the increasing stage until the end of the decreasing stage; and $R D$, the rate decreasing period.

Let's define $p$, to be the probability that upon arrival at the queue, the sampling cell will observe that the queue is under the threshold $T_{1}$, and therefore allow the source to increase its send rate. Approximation of $p_{1}$ is given in the appendix. We assume that all sources observe the same queue distribution upon the arrival of their respected sampling cells at the bottleneck queue. This assumption is reasonable considering that the bottleneck has very high utilization; the current sampling cell will observe the queue behavior that is resulted from the total traffic arrival at the bottleneck queue since the last sampling cell, including those that are from the corresponding source with the current sampling cell, minus the total traffic departed; and all sources are in the same class of users, that is they all run the same ARB algorithm.

For a given source ith with sampling interval $M s_{i}$, its average of the number of sampling cells in the rate increasing period per queue cycle 
is $R I / M s_{i}$. The average number of sampling cells in one cycle time CT from source $i$ is $C T / M s_{i}$. The probability of a sampling cell that actually results in increasing the send rate (note that the source will restraint from increasing with probability of $1-p_{l}$ ) per cycle, and therefore in general, is:

$P_{I}(I)=p_{l} \frac{R I}{C T}$.

In computing $P_{i}(D)$, the probability of a sampling cell that results in decreasing the send rate of source $i$, it is important to note that ARB sources will only cut once (they reset $D_{n}$ after a cut) upon detection of the queue during the $R D$ period. That is even if a source subsequent sampling cells arrive within $R D$ after a cut, it will not reduce its send rate. The maximum total number of rate reductions during $R D$ is therefore $\mathrm{N}$, one from each source. The probability that a sampling cell from source $\mathrm{i}$ will result in a cut per cycle, and so in general, is therefore:

$P_{i}(D)=\frac{\min \left(1, \frac{R D}{M s_{i}}\right)}{\frac{C T}{M s_{i}}}$.

The analysis given above yields upperbound analysis in that the standard deviation is used in all critical values of the time dependent model of ARB. It allows us to study the throughput behavior over short intervals of time where the upperbound conditions can occur (e.g., $\lambda_{\max }$ and $Q_{R}$ are high), and to compute the $T_{3}$ threshold. It is also useful to study the throughput ratio over a long period of time in steady state, and that requires the mean value analysis. This is done by suppressing the standard deviation in computing the ARB model equations described above. Also, from the analysis above, it is clear that there are different ARB parameters that can be used to effectively control the behavior of an ARB system as described in details in (Huynh, 1996).

The setting of $T_{3}$ threshold is for detecting when an ARB system is out of its normal operating range (e.g., abnormal conditions). For instance, when new connections are started or sudden bursts of higher priority traffic (e.g., time delay sensitive traffic) enter the network while the network is already saturated. When this condition happens, ARB will slow down significantly (SLOWDOWN2) to bring the network back to the normal operating range. As mentioned above, the upperbound analysis can be used to find an appropriate value of $T_{3}$ that has very small probability of an ARB system exceeding it during normal operation. 


\subsection{Conditions for Proportional Bandwidth Allocations}

From the above analysis, we form two conditions that must be satisfied in order to achieve proportional bandwidth allocation. These conditions that should be evaluated according to their ordering are described in the following.

\section{Condition 1}

From eq.(1), in order to guarantee that sources will get their allocated share of the bandwidth, the ratio of $P_{i}(I) / P_{i}(D)$ must be equal, and that requires either one of the two following subconditions to be satisfied: 1) sources must have the same sampling interval; or 2) the rate decreasing period, RD, must be less than or equal to the minimum sampling interval, $R D \leq \min \left(M s_{1, \ldots}, M s_{N}\right)$ (see Huynh, 1996).

\section{Condition 2}

In order to satisfy condition 2, either one of the following must hold: 1) sources have the same sampling interval; or 2) if sources have different sampling intervals, then $P_{D}+r t t \leq \min \left(M s_{1, \ldots}, M s_{N}\right)$.

There are different ARB parameters that can be used to effectively control whether or not an ARB system satisfies the two conditions required to provide proportional bandwidth allocation. They are the increment factor $\Delta l_{i}$, the decrease factor $\theta_{1}$, and the $T_{2}$ threshold, and the sampling interval Ms respectively (see Huynh, 1996 for an analysis of each of them).

\subsection{WORST CASE THROUGHPUT ALLOCATION}

We will only provide a summary of results in this section, please refer to (Huynh, 1996) for the analysis. The following results are worst case throughput allocation when condition 1 , or condition 2 , or both of the proportional throughput allocation described above are not satisfied.

Let us consider two sources, $i$ and $j$, respectively; and let source $i$ have shorter sampling period than source $j$. In the case where condition $\# 1$ is not satisfied such that $M s_{i}<R D<M s_{j}$, and condition \#2 is satisfied, that is $P_{D}+r t t \leq M s_{i}$, the ratio of the throughput between source $i$ and source $\mathrm{j}$ can be derived as follows:

$$
\frac{A_{i}}{A_{j}}=\frac{\Delta l_{i}}{\Delta l_{j}} \frac{R D}{M s_{i}} .
$$

In the case where condition 1 is met and condition 2 is not, the throughput ratio between source $i$ and source $j$ is given as:

$$
\frac{A_{i}}{A_{j}}=\frac{\Delta l_{i}}{\Delta l_{j}}\left[1+\frac{1}{p_{l}} \frac{P_{D}+r t t-M s_{i}}{R I}\right] .
$$


Next, we will consider the worst case scenario where both condition $\# 1$ and condition \#2 are not met. By combining the equations (4) and (5) above, we have the following worst case ratio of throughput allocation between source $i$ and source $j$ :

$$
\frac{A_{i}}{A_{j}}=\frac{\Delta l_{i}}{\Delta l_{j}}\left[1+\frac{1}{p_{l}} \frac{P_{D}+r t t-M s_{i}}{R I}\right] \frac{R D}{M s_{i}} .
$$

\subsection{NUMERICAL AND SIMULATION EXAMPLE}

Due to limited space, we only show a simple configuration and discuss briefly the results. Please refer to (Huynh, 1996) for more examples and detailed analysis. In the appendix, we provide approximations of important ARB model parameters that are used to calculate our analytical results.

\subsection{Case 1.a}

In Figure 2, there are two campus networks, A and B respectively, connected through a WAN. There are 24 sending stations attached to the Hub/Switch $A$ and 24 receiving stations attached to Hub B. Data are sent from stations in A to stations in B. The stations are split into two groups: group 1 consists of 8 stations (i.e., connections 1 through 8 ), and group 2 consists of the remaining 16 (i.e., connections 9 through 24). Connections in group 1 are allocated a throughput that is twice that of the connections in group 2.

For our first run in this configuration, the parameters are set as follows. The T1, T2, and T3 thresholds are set at 1 cell, 20 cells, and 160 cells respectively. The unit of increment, $R_{\text {inc }}$, is set to $0.2 \%$ of the channel capacity, i.e., $R_{\text {inc }}=0.002 \times 9 \mathrm{Mbps}=18 \mathrm{Kbps}$. Group 1 connections have an increment step of $\Delta l_{i}=2 \times R_{\text {inc }}$ for $i=1, \ldots, 8$. Group 2 connections have an increment step of $\Delta l_{i}=R_{\text {inc }}$ for $i=9, \ldots, 24$. The sampling interval is set to $30 \mathrm{~ms}$ for group 1 connections, and $60 \mathrm{~ms}$ for group 2 connections respectively. The cut factor $\theta_{1}$ is set to 0.9 .

\section{Upperbound Analysis}

Computation of the ARB model parameters yield the following: The resulting maximum rate is $\lambda_{\max }=9.282 \mathrm{Mbps}$; the length of the rate decreasing period is $R D=0.048 \mathrm{sec}$, the roundtrip time is approximately $0.01 \mathrm{sec}$; the minimum total send rate is $\lambda_{\min }=8.45 \mathrm{Mbps}$; the length of the total queue decreasing stage is $P_{D}=0.051 \mathrm{sec}$; and the length of the rate increasing period is $R I=0.0785 \mathrm{sec}$. Since both conditions are not satisfied, the worst case throughput ratio between group 1 and group 2 from this upperbound analysis is, based on eq. (6), is 4.8 instead of 2 as their allocated ratio.

In using the upperbound analysis for computing the $T_{3}$ threshold, we want to obtain a threshold value such that over $99 \%$ of the time, the 


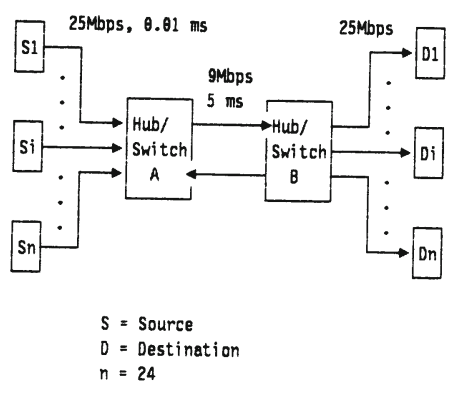

Figure 2. Proportional Bandwidth Allocation Configuration

maximum queue will be below this value. The computation yields a value of 45 cells for $T_{3}$.

\section{Mean Value Analysis}

Based on the mean value analysis, we obtain the following values: $\lambda_{\max }=9.2 \mathrm{Mbps}, R D=0.035 \mathrm{sec}, \lambda_{\min }=8.47 \mathrm{Mbps}, P_{D}=0.041 \mathrm{sec}$, and $R I=0.07 \mathrm{sec}$. Again, even with the mean value analysis, the length of $R D$ violates the condition 1 of proportional bandwidth allocation since it is larger than the sampling interval of $30 \mathrm{~ms}$ for the group 1 connections. Condition 2 is also violated. The worst case throughput ratio between group 1 and group 2 is therefore 3.28 instead of 2 .

\section{Simulation}

Figure 3 shows the total send rate and the queue behavior at the 9 Mbps channel. Figure 4 shows the average throughput claimed by each of the connection in each group. As expected from both the upperbound and the mean value analysis, the throughput does not follow the 2 to 1 ratio that is allocated to each of the connection group. This ratio is approximately 3.2 compared with 3.28 from the mean value analysis, and 4.8 from the upperbound value analysis. Also, as expected from the upperbound analysis, no difference is observed in the final results of separate simulation runs when the upper threshold $T_{3}$ value is set to 160 cells and 45 cells respectively.

\subsection{Case 1.b}

For this run, we will change the ARB parameters, mainly the rate increments and the $T_{2}$ threshold, so that the proportional bandwidth allocation conditions will be satisfied. The following parameters are changed from case 1.a: the $T_{1}$, and $T_{2}$ thresholds are set at 1 cell, and 5 cells respectively; $R_{\text {inc }}=0.001 \times 9 \mathrm{Mbps}=9 \mathrm{Kbps}$. 
ADAPTIVE RATE-BASED CONGESTION CONTROL

TOTAL SEND RATE AND QUEUE BEHMOR

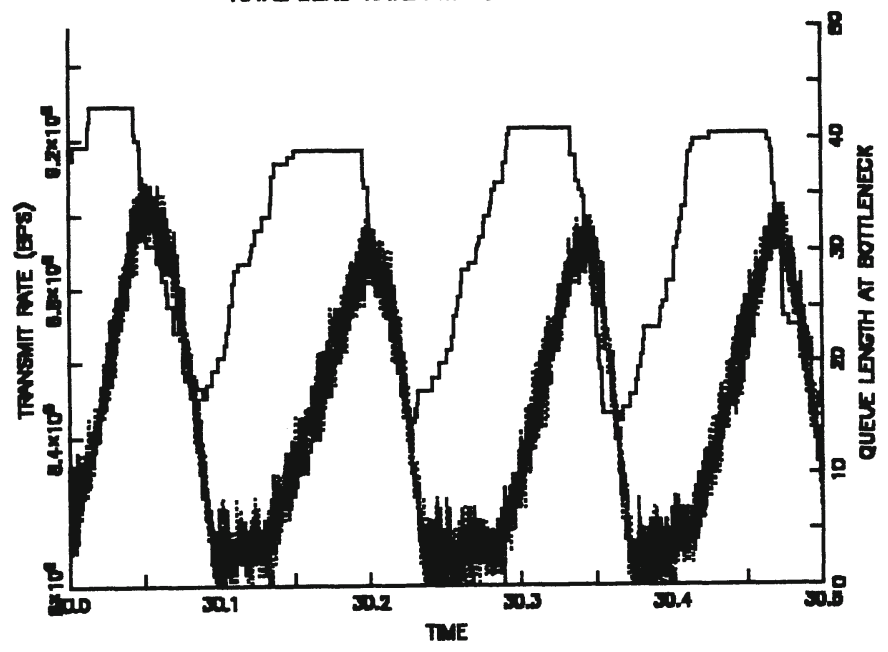

Figure 3. Total send rate and queue behavior at the $9 \mathrm{Mbps}$ channel

\section{Upperbound Analysis}

The model parameters are obtained as follows: $\lambda_{\max }=9.177 \mathrm{Mbps}$, $R D=0.039 \mathrm{sec}, P_{D}=0.03 \mathrm{sec}, \lambda_{\min }=8.425 \mathrm{Mbps}$, and $R I=0.144 \mathrm{sec}$. The upperbound analysis still shows that both conditions are not satisfied. Therefore, we may expect that over some short intervals where upperbound conditions exist, the throughput will not follow the desired ratio. The worst case ratio if the upperbound conditions do exist over a period of time is 2.85 instead of the desired ratio of 2 .

As in case 1.a above, our computation yields a value of 23 cells that can be used in setting $T_{3}$.

\section{Mean Value Analysis}

Using the mean value analysis, we obtain the following values: $\lambda_{\max }=9.12 \mathrm{Mbps}, R D=0.0276 \mathrm{sec}, P_{D}=0.023 \mathrm{sec}, \lambda_{\min }=8.5 \mathrm{Mbps}$, and $R I=0.118 \mathrm{sec}$. Interestingly, the mean value analysis shows that the length of $R D$ is less than the sampling interval of group 1 connections and therefore satisfies condition \#1 of the proportional bandwidth allocation. For condition \#2, the sum of $P_{D}+r t t=0.033 \mathrm{sec}$ is still larger than the minimum sampling interval of $0.03 \mathrm{sec}$ in our configuration. This is less than ideal but is still tolerable. The worst case throughput allocation ratio is 2.07 instead of the desired 2. Therefore, we can declare that condition \#2 is also satisfied. 


\section{ADAPTIVE RATE-BASED CONGESTION CONIROL}

SEND RATE OF GROUP1 AND GROUP2 CONNEETIONS

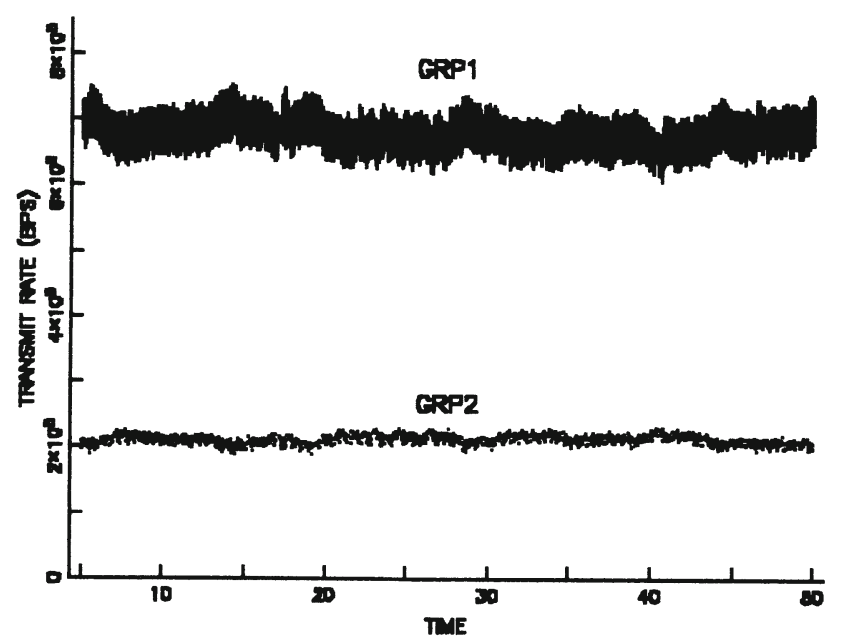

Figure 4. Average throughput for each connection in the groups (case 1.a)

\section{Simulation}

Figure 5 shows the simulation result of the average send rates of each group for case 1.b. The average throughput of each group over the simulation run follows the desired ratio of 2 to 1 (with 2 for group 1 connections). Also, as expected from the upperbound analysis, no difference is observed in the final results of separate simulation runs when the upper threshold $T_{3}$ value is set to 160 cells and 23 cells respectively.

\subsection{FUTURE WORK}

In high speed environment, it is desirable to take advantage of the available bandwidth as quickly as possible to minimize the time that resources are not utilized efficiently. The rate increment used in this paper is small to avoid large oscillations when the network is saturated. But the analysis suggests that the algorithm can learn when the network is not in saturation and therefore can have larger increment to take advantage of the available bandwidth in the network.

The type of traffic that we studied in this paper is homogeneous in that all connections sharing a bottleneck link are ARB type connections, that is they're all controlled by the ARB algorithm (e.g., having similar Quality of Service, QOS). Clearly, we should be able to mix other type of traffic with ARB, e.g., higher priority CBR/VBR traffic. Preliminary simulation studies show that the proportional bandwidth allocation works very well even in the presence of VBR traffic. 


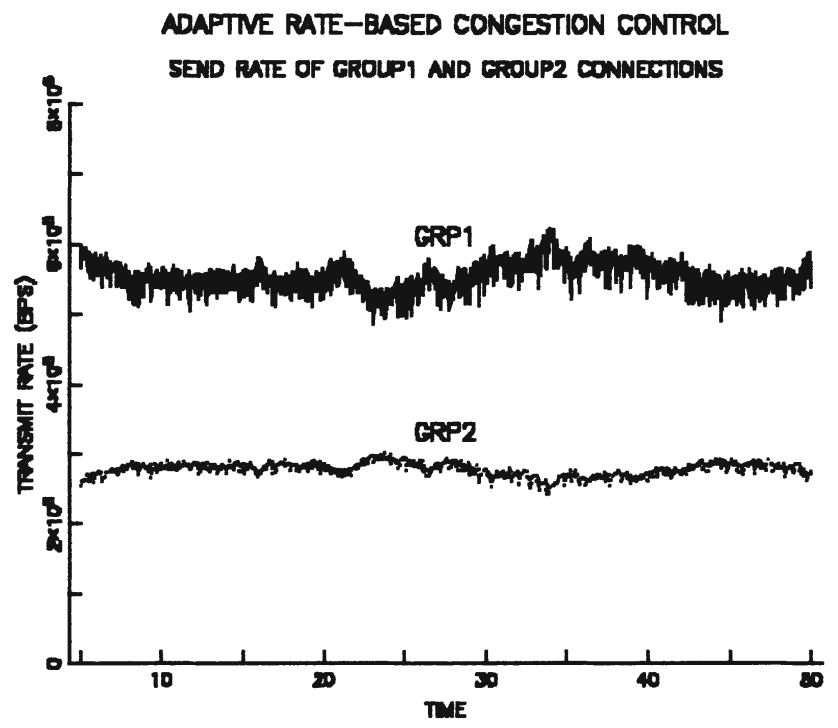

Figure 5. Average throughput for each connection in the groups (case 1.b)

\subsection{APPENDIX}

\subsection{The Rate Increasing Factor}

Let $q_{i, n}$ be the queue length seen by source i's sampling cell at the $n$th epoch. Let $x_{n}$ be the number of arrivals and $y_{n}$ be the number of cells served during source i's $n$th sampling interval, $M s_{i}$, respectively.

The queue seen by source $i^{\prime} s \quad n+1^{\text {st }}$ sampling cell is: $q_{i, n+1}=q_{i, n}+x_{n}-y_{n}$ An assumption is made here in that the server at the bottleneck is always busy (an assumption for the ARB model). The delay change observed by source $i^{\prime} s n+1^{\text {st }}$ sampling cell is therefore: $q_{i, n+1}-q_{i, n}=x_{n}-y_{n}$.

Clearly, the number of cells served $y_{n}$ is fixed and is equal to $\frac{M s_{i}}{C_{t}}$, where $c_{t}$ is the cell transmission time. The number of arrivals, $x_{n}$, consists of two parts, a deterministic part and a random number of part. The deterministic part comes from the periodic nature of the sources, and the random part comes from the sampling interval $M s_{i}$ relative to the source j's period $s_{j}$ (i.e., $s_{j}$ is just 1 over the send rate of source $j$ during source i's $n$th sampling interval. This send rate can increase and so the period $s_{j}$ can change, but this change is small due to the small rate increment, it is negligible.)

The deterministic part of arrival from source $j$ during source $i$ 's $n$th sampling interval is $\mathrm{I}\left[\frac{M s_{i}}{s_{i}}\right]$, and the random part can be derived as shown in section 3 . The totat arrival, $x_{n}$, is therefore: 


$$
x_{n}=\sum_{j=1}^{N} \mathrm{I}\left[\frac{M s_{i}}{s_{j}}\right]+\sum_{\substack{j=1 \\ j=i}}^{N} r_{j}
$$

Let $R_{n}$ be the random part of the total arrival $x_{n}$, then $R_{n}$ has a binomial distribution. The variance of $R_{n}$ has an upper bound of $\frac{1}{4}(N-1)$ (i.e., $\left.\operatorname{Var}\left(R_{n}\right) \leq \frac{1}{4}(N-1)\right)$.

${ }^{4}$ During the rate increasing period, the mean queue length is small and less than the $T_{1}$ threshold, even when the utilization is high, that is $\frac{\bar{x}_{n}}{y_{n}}=\frac{d_{n}+E\left(R_{n}\right)}{\text { mean of the random part of } x_{n} \text { respectively. Any fluctuations in the }}$ queue is mainly due to the random part of the total arrival $x_{n}$.

By using the normal distribution to approximate the binomial distribution of $R_{n}$, we can obtain the average probability of rate increase as follows:

$$
\begin{aligned}
p_{l} & \simeq P\left[R_{n} \leq E\left(R^{n}\right)+T_{1}\right] \\
& \simeq \mathbb{N}\left(\left(T_{1}+\frac{1}{2}\right) h\right) .
\end{aligned}
$$

Where $h=1 / \sqrt{0.25(N-1)}$, and $\mathbb{N}(x)$ is the normal distribution function $(0,1)$. Next, We will use the approximation obtained from above for $p_{1}$ to derive the increasing factor $\alpha$. As described in section 2, let the rate increment of source $i$ be $\Delta l_{i}$. At each sampling epoch during the rate increasing period $(R I)$, source i can increase its send rate with probability $p_{1}$. As a result, source i contributes to the total rate increasing factor $\alpha$ by an amount of $p_{1} \frac{\Delta l_{i}}{M s_{i}}$. The total contribution by all
the sources are thus:

$$
\alpha=p_{I} \sum_{i=1}^{N} \frac{\Delta I_{i}}{M s_{i}} \text {. }
$$

\subsection{The Rate Decreasing Factor}

In the following, we will derive the rate decreasing factor, $\beta^{t}$. Let's again split the $N$ sources into $K$ classes and let $\lambda_{k}$ be the total mean arrival rate, and $M s_{k}$ be the sampling interval of sources in class $k$ respectively, $k=1, \ldots, K$. As mentioned before, each source will cut only once during the decreasing stage so the total cut from class $\mathrm{k}$ is $\theta_{1} \lambda_{k}$. This total cut distributes exponentially over the sources' sampling interval $M s_{k}$. In other words, the rate reduction from sources in class $\mathrm{k}$ 
is: $\quad \theta_{1}\left[\frac{t}{M s_{k}}, 1\right]^{-} \lambda_{k}$, where $(x, y)^{-}=\min (x, y)$. The total rate reduction from all the sources is thus equal to: $\sum^{k} \theta_{1}\left[\frac{t}{M s_{i}}, 1\right]^{-} \lambda_{i}$.

By replacing $\lambda_{i}$ with a portion of $\lambda_{\max }$, that is allocated to class $\mathrm{i}$ sources, and let $f_{i}$ be this portion, then we have the following:

$$
\begin{aligned}
\beta^{t} & =\sum_{i=1}^{k} f_{i} \theta_{1}\left[\frac{t}{M s_{i}}, 1\right]^{-} \\
& =\sum_{i=1}^{k} f_{i} \beta_{i}\left(t, M s_{i}\right)^{-}
\end{aligned}
$$

Where $\beta_{i}=\theta_{1} \frac{1}{M s_{i}}$ Although this $\beta^{t}$ function is a sum of exponential factors of similar form (i.e., $\beta_{i}^{t}$ for $i=1, \ldots, K$ ), it would not change the result obtained for the queue behavior during the decreasing stage in section 3. In fact, we can replace the term $\frac{\beta^{t}-1}{\ln \beta}$ with the sum above:

$$
\frac{\beta^{t}-1}{\ln \beta}=\sum_{i=1}^{K}\left[\frac{\beta_{i}^{\left(t, M s_{i}\right)^{-}}-1}{\ln \beta_{i}}\right] f_{i} \text {. }
$$

\subsection{REFERENCES}

Altman, E. and Baccelli, F. and Bolot, J.C. (1994) Discrete Time Analysis of Adaptive Rate Control Mechanisms. IFIP Transactions on Communications, Communication Systems (Netherlands), Volume C, No. 21.

Artiges D. (1994) Stability Analysis of an Adaptive Rate Based Congestion Control Algorithm. NCSU Research Report.

ATM Forum Technical Committee (1996) Traffic Management Specification Version 4.0. ATM Forum/95-0013R10 (February).

Fratta, L. and Musumeci, L. (1995) Performance Evaluation of FR, SMDS, and ABR Services in ATM Networks. Proceedings of Sixth IFIP WG 6.3 Conference on Performance of Computer Networks (October).

Haas, Z. (1991) Adaptive Admission Congestion Control. ACM Computer Communications Review, Volume 21, No. 5 (October).

Huynh, L. (1996) Adaptive Rate-Based Congestion Control And

Proportional Bandwidth Allocation In High-Speed Networks. Phd thesis, North Carolina State University.

Jacobson, V. (1988) Congestion Avoidance and Control. ACM SIGCOMM'88 (August).

Jain, R. (1989) A Delay-Based Approach for Congestion Avoidance in Interconnected Heterogeneous Computer Networks. ACM SIGCOMM'89, Volume 19, No. 5.

Jain, R. (1992) Myths About Congestion Management in High-Speed Networks. Information Network and Data Communication IV, Elsevier Science, Publishers B.V. (North-Holland).

Keshav, S. (1991) A Control-Theoretic Approach to Flow Control. Computer Communication Review (USA), Volume 21, No. 4 (September). 
Kim, H. and Farber, D.J. (1995) A New Congestion Control Framework for Large Bandwidth-Delay Product Networks. Proceedings of HPN VI, IFIP 6th International Conference on High Performance Network (September).

Kolarov, A. and Ramamurthy, G. (1995) End-to-End Adaptive Rate Based Congestion Control Scheme for ABR Service in Wide Area ATM Networks. ICC'95 (June).

Mitra, D. and Seery, J.B. (1991) Dynamic Adaptive Windows For High-Speed Data Networks With Multiple Paths and Propagation Delays (Extended Abstract). IEEE INFOCOM'91, Volume 1 (April).

Mukherjee, A. and Strikwerda, J. (1994) Analysis of Dynamic Congestion Control Protocols -- A Fokker-Planck Approximation. Journal of High Speed Networks (Netherlands), Volume 3, No. 1.

Newell, G.F. (1982) Applications of Queueing Theory. Second Edition, Chapman and Hall.

\subsection{BIOGRAPHY}

Lap T. Huynh is an Advisory Engineer in the Networking Software Division at IBM, Research Triangle Park, North Carolina. Since he started at IBM, he has worked on various networking architectures and implementations. His work and interests are in areas of congestion control for high speed networks, performance analysis, and prototyping. He obtained a BS in Electrical Engineering from University of Washington, an MS in Computer Engineering from Florida Atlantic University, and a Ph.D. in Computer Engineering at North Carolina State University in 1983, 1987, and 1996 respectively.

Arne A. Nilsson received the M.S. and Ph.D. degrees from Lund Institute of Technology, Lund, Sweden in 1968 and 1976, respectively. From 1973 to 1978 he was an Assistant Professor in the Department of Electrical Engineering at Lund Institute of Technology. In 1978 he joined the Department of Electrical and Computer Engineering at North Carolina State University, Raleigh, North Carolina, where he is now a Professor. Since 1982 he has been the Program Manager for the Networking area in the Industry/University Center for Communications and Signal Processing at NCSU. He has also been Director and Technical Director of this research Center. From 1986 to 1987 he held the position of Professor at the Royal Institute of Technology, Stockholm, Sweden. He has published more than 100 papers in technical journals and conferences and has consulted for many government agencies and corporations. 